\title{
Cryogenic infrared optics for the SPICA coronagraph
}

\author{
Keigo Enya ${ }^{1}$, Takao Nakagawa ${ }^{1}$, Hirokazu Kataza ${ }^{1}$, \\ Hidehiro Kaneda ${ }^{1}$, Yukari Yamashita Yui ${ }^{1}$, Motohide Tamura ${ }^{2}$, \\ Lyu Abe ${ }^{2}$, Yoshiyuki Obuchi ${ }^{2}$, Takashi Miyata ${ }^{3}$, Shigeyuki Sako ${ }^{3}$, \\ Takashi Onaka ${ }^{4}$, Hidenori Takahashi ${ }^{4}$ and the SPICA team \\ ${ }^{1}$ Department of Infrared Astrophysics, Institute of Space and Astronautical Science, Japan \\ Aerospace Exploration Agency, 3-1-1 Yoshinodai, Sagamihara, Kanagawa, 229-8510 \\ ${ }^{2}$ Optical and Infrared Astronomy Division, National Astronomical Observatory of Japan, \\ 2-21-1 Osawa,Mitaka, Tokyo, 181-8588 Japan \\ ${ }^{3}$ Institute of Astronomy, University of Tokyo, 2-21-1 Osawa, Mitaka, Tokyo, 181-8588 Japan \\ ${ }^{4}$ Department of Astronomy, University of Tokyo, 7-3-1 Hongo, bunkyo, Tokyo, 113-0033 Japan \\ email: enya@ir.isas.jaxa.jp
}

\begin{abstract}
SPICA is a cooled, single large-mirror space-telescope, which is under discussion as an succsesor of the ASTRO-F mission. One of the most ambitious challenges of the SPICA mission is the direct observations of exoplanets with a coronagraph instrument. We report cryogenic infrared optics to realize high quality wavefronts for the SPICA coronagraph.

The SPICA satellite will be launched by an H-IIA rocket to Sun-Earth L2 Halo orbit early in the 2010s. The SPICA telescope is a Ritchey-Chretien optics with $3.5 \mathrm{~m}$ diameter primary mirror, and cooled down to $4.5 \mathrm{~K}$ in orbit by radiation cooling and mechanical cryo-coolers. Main working wavelengths are 5-200 micron. Advantages of the SPICA coronagraph are the infrared wavelenths where the contrast between planets and central stars are smaller than the optical wavelengths, and that the cooled space telescope consists of monolithic mirrors.

Development of light-weight cooled telescope is one of the most important tasks to realize SPICA. At the present, sintered $\mathrm{SiC}$ and carbon fiber reinforced $\mathrm{SiC}(\mathrm{C} / \mathrm{SiC})$ composite are candidate materials for the mirrors, truss, and optical bench. For these materials, estimations and improvements of basic property and surface roughness in cryogenic temperatures have been carried out. Deformation of trial product mirrors by cooling is also examined.

We are developing cryogenic deformable mirrors (DMs) because wave front accuracy of the SPICA telescope is 0.35 micron RMS, which is not enough for our coronagraphic instrument. For MEMS (Micro Electro Mechanical System) DM and some others, measurements of thermal deformation by cooling, electrical response, and heat generation are undergoing. Developments of a tip-tilt system for cryogenic usage started to cancel vibration caused by the cryo-coolers and other components and to realize a diffraction limit resolution. The first result of our binary mask coronagraph experiment is also shown.
\end{abstract}

Keywords. SPICA, infrared, telescope, cryogenic, tip-tilt, deformable mirror.

\section{Outline of the SPICA mission}

The SPICA (SPace Infrared telescope for Cosmology and Astrophysics) is the third Japanese infrared space-borne project (Nakagawa 2004, Onaka et al. 2005) following the Infrared Telescope in Space (IRTS) and ASTRO-F which will be launched in 2006 (Murakami 2004). SPICA is equipped a telescope with $3.5 \mathrm{~m}$ aperture consists of a monolithic mirror. The whole telescope is cooled down to $4.5 \mathrm{~K}$ by radiation cooling and mechanical cryo-cooler. Required optical quality of the telescope is diffraction limited at 

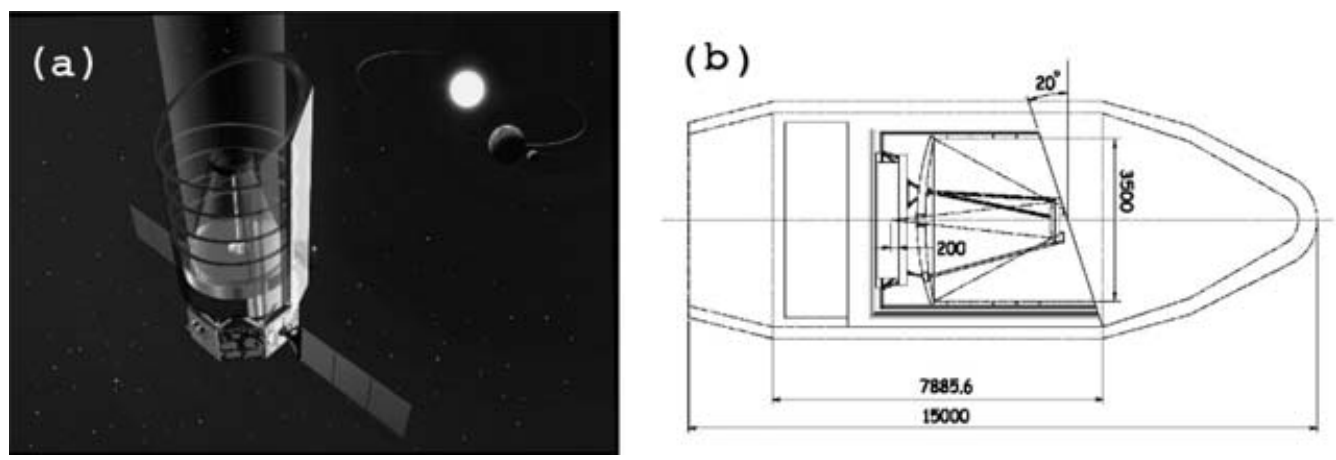

Figure 1. (a):Image of the SPICA satellite in orbit. (b):Illustrative view of the SPICA telescope in the H-IIA rocket fairing.

5 micron wavelength. Main observing wavelengths are 5-200 micron, therefore SPICA is complementary to James Webb Space Telescope (JWST)(Sabelhaus 2004). and Herschel Space Observatory (HSO)(Pilbratt 2004). SPICA will be launched into the Sun-Earth libration $\mathrm{L}_{2}$ halo orbit by the H-IIA rocket early in the 2010s and operated for 5 years.

A coronagraph instrument will be equipped with other general purpose instrumetns on SPICA (Tamura et al. 2005, Abe et al. 2005). SPICA is a unique opprotunity because of its infrared observation with a large aperture and simple pupil provided by a monolithic mirror.

\section{Develop of the telescope}

\subsection{Design and material}

The SPICA telescope is a Ritchey-Chretien optics, consisting of two monolithic mirrors with $3.5 \mathrm{~m}$ aperture and is cooled down to $4.5 \mathrm{~K}$ before operation.

At the present moment, sintered $\mathrm{SiC}$ (figure $2 \mathrm{a}, \mathrm{b}$ ) and carbon fiber reinforced $\mathrm{SiC}$ $(\mathrm{C} / \mathrm{SiC}$ ) composite (figure $2 \mathrm{c}, \mathrm{d}$ ) are candidate materials for the SPICA mirror, truss and optical bench (Enya et al. 2004). These materials have some advantages, especially their high stiffness to make light weight mirrors.

Sintered $\mathrm{SiC}$ is used in HSO and other missions, therefore this material is superior in their actual results. However, brittleness is a disadvantage of $\mathrm{SiC}$. Improved $\mathrm{C} / \mathrm{SiC}$ composite is superior in its fracture toughness. SiSiC slurry coating technique reduces surface roughness of the $\mathrm{C} / \mathrm{SiC}$ composite mirror.

\section{2. $\phi 700 \mathrm{~mm} C / \mathrm{SiC}$ composite BBM mirror}

In the SPICA mission, one of the most important properties of the mirror is not to deform its figure in cooling down to 4.5K. We developed a BBM spherical mirror (Nakagawa 2004, Onaka et al. 2005) made of C/SiC composite with SiSiC slurry coating (figure 3a, b). The mirror consists of 6 segments to demonstrate feasibility of joint technology for fabrication of $3.5 \mathrm{~m}$ diameter mirror. Cryogenic test of the mirror was executed with cryo-chamber (figure 3c) that was the same one with tests of the ASTRO-F telescope (Kaneda et al. 2005) cooled by liquid Nitrogen and Helium. The surface figure was monitored by Zygo GPI-XP. The result are shown in (figure 3d, e). Significant deformation by cooling was confirmed. 


\section{Sic telescope}

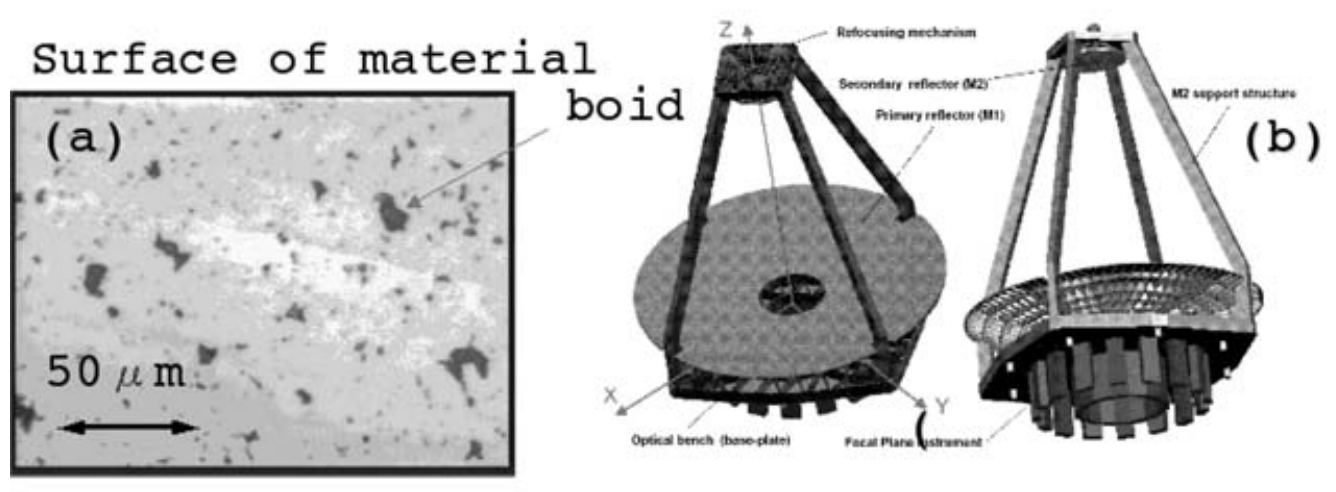

\section{C/SiC composite telescope}

Surface of material

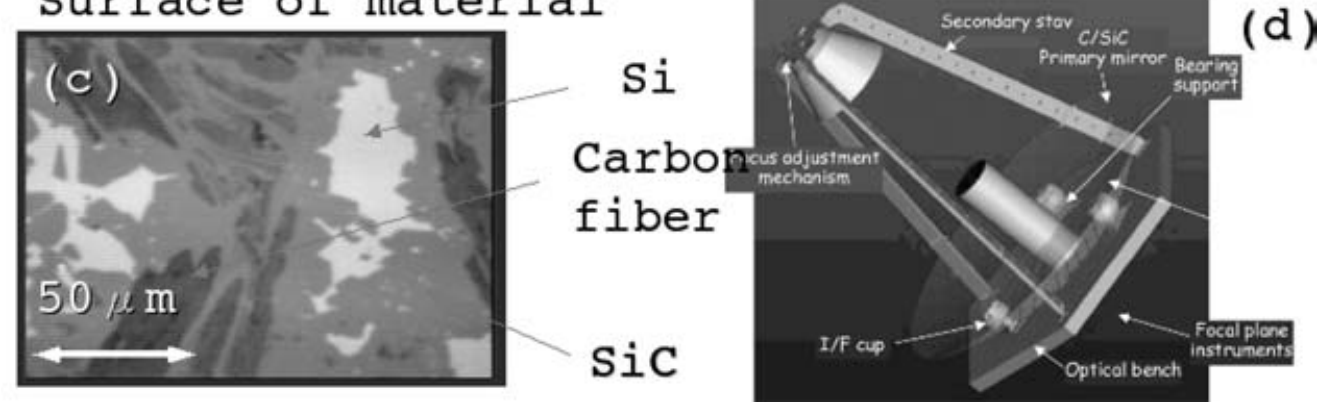

Figure 2. Designs of the SPICA telescope. Surface of polished Sintered SiC (a) and a telescope design with sintered $\mathrm{SiC}(\mathrm{b})$. Surface of polished bare C/SiC (c) composite and a telescope design with this material (d).

\section{Cryogenic tip-tilt mirror system}

\subsection{Why is it required?}

Pointing error in SPICA caused by vibration of cooler, compressor, and other components can be more than 1 arcsec, if any provisions are applied. On the other hand, pointing accuracy to be 0.03 arcsec is required for the coronagraph design. This gap is too large to be solved by improvement of pointing system. Therefore, a cryogenic tip-tilt mirror system is indispensable.

\subsection{Development and experiment}

Design of proto-type of a cryogenic tip-tilt mirror is shown in figure 4(a). Figure 4(b) shows the cryogenic chamber cooled down to $10 \mathrm{~K}$ with GM cryo-cooler. Motion of a cryogenic piezo actuator was confirmed in this chamber. The system also demonstrated mirror chopping in room temperature. A proto-type system is now under construction and examination.

It turned out that heat generation is a critical problem (10 times larger than specification of less than a few $\mathrm{mW}$ ). We are going to test selection of actuators and adoption of support with a lever mechanism to reduce the heat generation. 

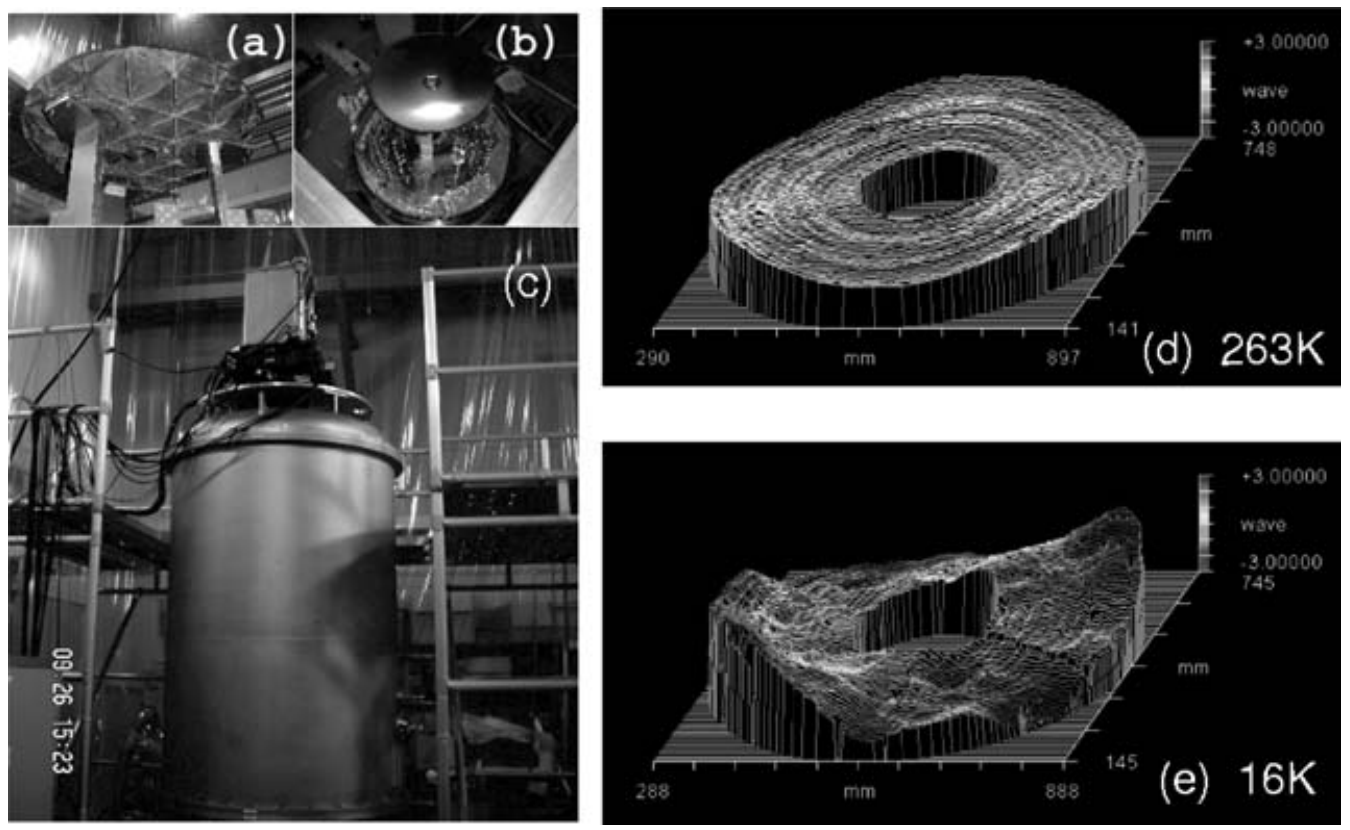

Figure 3. Cryogenic test of the $\phi 700 \mathrm{~mm} \mathrm{C} / \mathrm{SiC}$ composite BBM mirror. The mirror (a), (b) and the cryogenic chamber for the test (c). (d) and (e) show thermal deformation of surface figure. Here figure of ambient temperature was subtracted and power was corrected. RMS of (d) and (e) are $0.40 \lambda$ and $1.21 \lambda$ respectively where $\lambda=632.8 \mathrm{~nm}$.
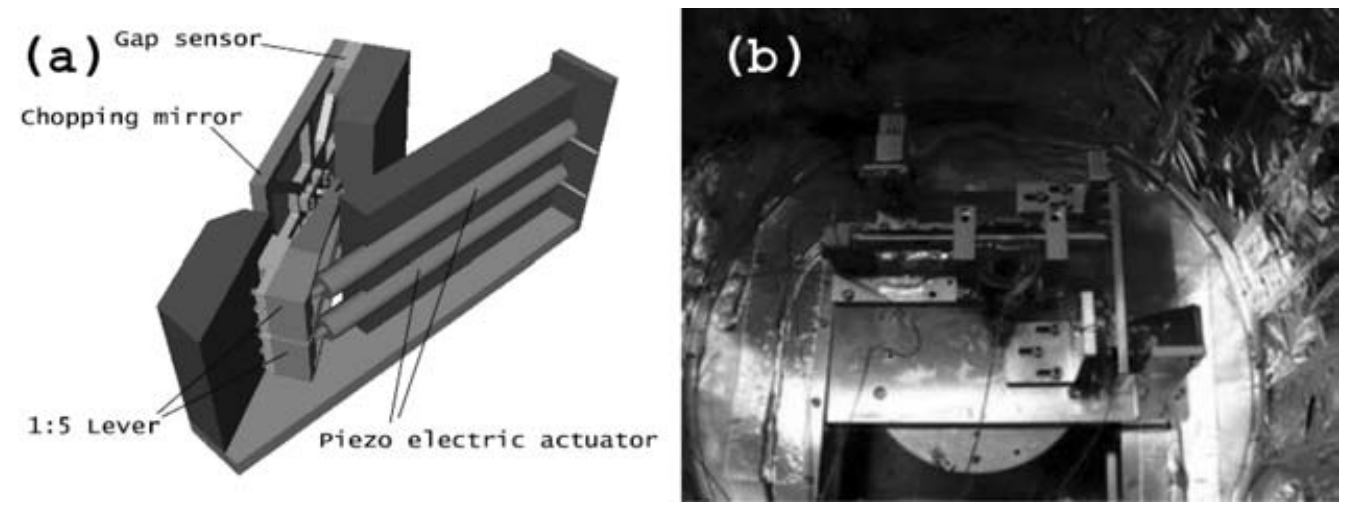

Figure 4. (a): Design of a proto-type of cryogenic tip-tilt mirror. (b): Piezo actuator set in a dewar with a $4 \mathrm{~K}$ cryo-cooler. Normal motion was confirmed at cryogenic temperature, while its stroke was one order smaller than in ambient temperature.

\section{Cryogenic deformable mirror}

\subsection{Why is it required?}

Specification of wave front error of the SPICA telescope is 0.35 micron RMS, while the coronagraph requires $\sim 10 \mathrm{~nm}$ order RMS. This gap is too large to be solved by improvement of the telescope. Therefore, cryogenic deformable mirror (DM) is indispensable for the SPICA coronagraph.

The mirror of figure 5(a) is a MEMS DM with $2 \mathrm{~mm}$ clear aperture and 32 channel actuators producted by BMC (Boston Micromachines Corporation). 

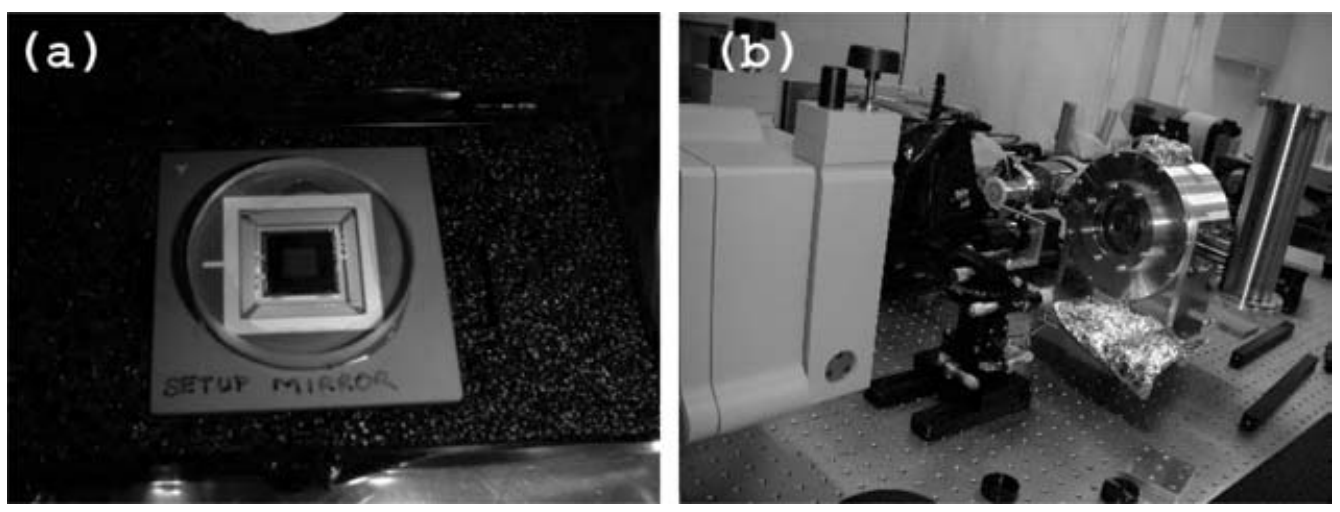

Figure 5. (a): 32 channel MEMS-deformable mirror producted by BMC (Boston Micromachines Corporation). (b): Set-up for cryogenic examination of DMs. This system consists of a ZYGO GPI-XP interferometer, cryo-chamber cooled by liquid nitrogen and zooming optics.

\subsection{Development and experiment}

Measurements of thermal deformation by cooling, electrical response, and heat generation are undergoing for some DMs (figure 5b). Zoomed surface figures of DMs in cryostat can be measured by Zygo GPI-XP. It turned out that deformation caused by difference of coefficients of thermal expansion between materials consisting of these devices is a critical problem. We are now developing a new configuration of substrate and adhesive in these devices to reduce the thermal deformation.

\section{Coronagraph experiments}

We think that binary shaped pupil mask is one of the most promising coronagraphic methods because of its achromatism, robustness against pointing error, and feasibility of mask fabrication ( Tanaka et al. 2005a, 2005b). We started our experiments of coronagraph with visible light (He-Ne laser with wavelength to be $632.8 \mathrm{~nm}$ ) but without any wave front control and vacuum chamber. Binary mask pattern of aluminum thin coating (thickness is 100nm) was made on a glass substrate by nano-fabrication technology usually used for productions of semi-conductor devices (figure 6a, b). The mask was a kind of checkerboard mask (Vanderbei et al. 2005) designed to avoid obstruction by the secondary mirror and spiders.

The first result of our binary mask coronagraph experiments is shown in figure 6(c), (d). The profile of figure 6(c) is along an arrow shown in figure 6(d). At present, the achieved contrast is only $10^{4}$, while the specification of contrast in this experiment is $10^{6}$. Improvement of the experiment and examinations of other coronagraphic methods should be executed. Ultimately, we will develop an infrared and cryogenic coronagraph for the SPICA mission. The newest results obtained after the conference is shown in Abe et al. 2005.

\section{Acknowledgements}

We would like to acknowledge useful comments of J. Nishikawa, N. Murakami, K. Fujita and Y. Itoh. We are also grateful to support the mask fabrication by National Institute of Advanced Industrial Science and Technology. This work was supported by a Grant-in Aids for Science Research from the Japan Society for the Promotion of Science. 

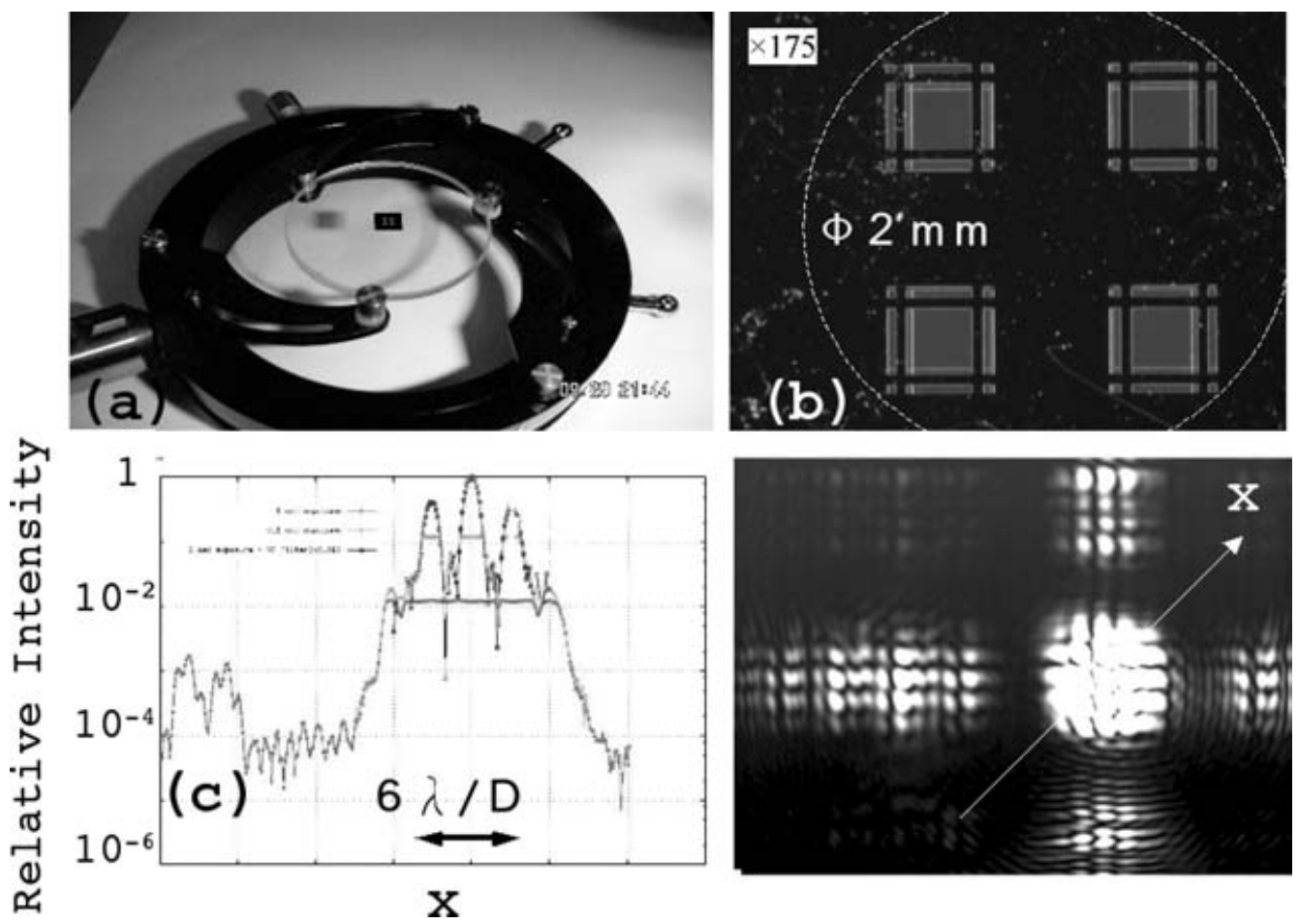

Figure 6. The first result of our coronagraph experiment made by nano-fabrication in visible light wavelength. $(\mathrm{a}, \mathrm{b})$ : The mask consist of aluminum with $100 \mathrm{~nm}$ thickness on a glass substrate. (c, d): Obtained profile and image of a point spread function respectively. Here lambda is wavelength of He-Ne laser $(632.8 \mathrm{~nm})$ and $\mathrm{D}$ is diameter of the beam to be $2 \mathrm{~mm}$.

\section{References}

Abe, L., Tamura, M., Enya, K.,Tanaka, S., Nakagawa, T., Kataza, H., Fujita,K., Nishikawa, J., \& Murakami, N. 2005, Proc. of IAUC200(this conference), submitted

Enya, K., Nakagawa, T., Kataza, H., Kaneda, H., Yamashita, Y. Y., Onaka, T., Oshima, T., \& Ozaki, T. 2004, Proc of SPIE 5487, 1092

Kaneda, H., Onaka. T., Nakagawa, T., Enya, K., Murakami, H., Yamashiro, R., Ezaki, T., Numao, Y., \& Sugiyama, Y. 2005, Applied Optics, submitted

Murakami, H. 2004, Proc. of SPIE5487, 330

Nakagawa, T. 2004, Advances in Space Research 34 , 645

Onaka, T. \& Nakagawa, T. 2005, Advances in Space Research, in press

Pilbratt, G. T. 2004, Proc. of SPIE 5487 , 401

Sabelhaus, P. A. \& Decker, J. 2004, Proc. of SPIE 5487, 550

Tanaka, S., Enya, K., Nakagawa, T., \& Kataza, H. 2005, PASJ, submitted

Tanaka, S., Nakagawa, T., Enya, K., Kataza, H., Tamura, M., Abe, L., and the SPICA coronagraph team 2005, Proc. of IAUC200(this conference), submitted

Tamura, M., Abe, L., HiCHAO team, SPICA coronagraph team \& JTPF working group 2005, Proc. of IAUC200(this conference), submitted

Vanderbei, R. J., Kasdin, N. J., \& Spergel, D. N. 2004, ApJ 615, 555 